\title{
Article
}

\section{Solid-State White Light-Emitting Electrochemical Cells Using Iridium-Based Cationic Transition Metal Complexes}

Hai-Ching Su, Hsiao-Fan Chen, Fu-Chuan Fang, Chih-Che Liu, Chung-Chih Wu, Ken-Tsung Wong, Yi-Hung Liu, and Shie-Ming Peng

J. Am. Chem. Soc., 2008, 130 (11), 3413-3419• DOI: 10.1021/ja076051e • Publication Date (Web): 27 February 2008

\section{Downloaded from http://pubs.acs.org on February 18, 2009}

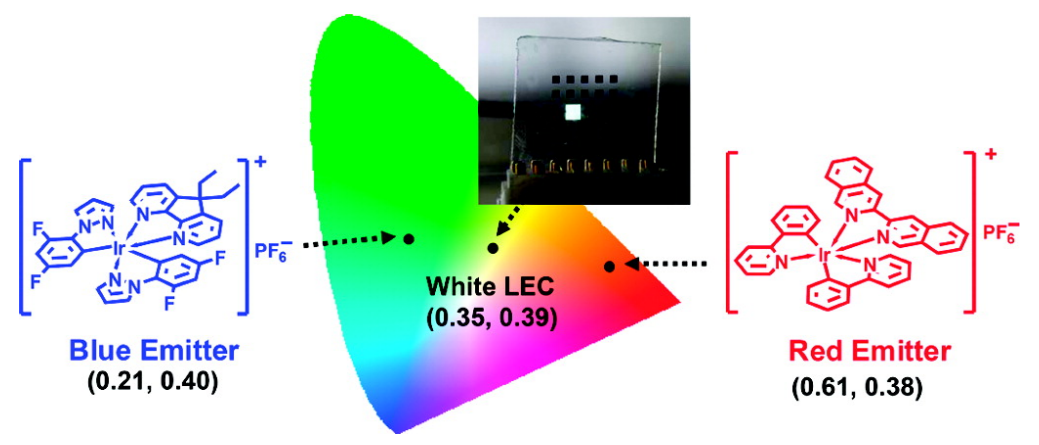

\section{More About This Article}

Additional resources and features associated with this article are available within the HTML version:

- $\quad$ Supporting Information

- $\quad$ Links to the 3 articles that cite this article, as of the time of this article download

- $\quad$ Access to high resolution figures

- $\quad$ Links to articles and content related to this article

- Copyright permission to reproduce figures and/or text from this article

\section{View the Full Text HTML}




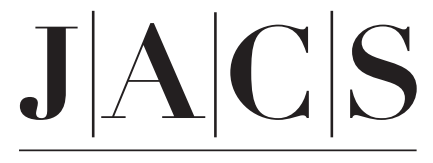

A R T I C L E S

Published on Web 02/27/2008

\title{
Solid-State White Light-Emitting Electrochemical Cells Using Iridium-Based Cationic Transition Metal Complexes
}

\author{
Hai-Ching Su, ${ }^{\dagger}$ Hsiao-Fan Chen, ${ }^{\ddagger}$ Fu-Chuan Fang, ${ }^{\ddagger}$ Chih-Che Liu, ${ }^{\dagger}$ \\ Chung-Chih Wu, ${ }^{*} \dagger$ Ken-Tsung Wong, ${ }^{*}, \ddagger$ Yi-Hung Liu, ${ }^{\ddagger}$ and Shie-Ming Peng ${ }^{\ddagger}$ \\ Department of Electrical Engineering, Graduate Institute of Electro-optical Engineering and \\ Graduate Institute of Electronics Engineering, National Taiwan University, Taipei 10617, \\ Taiwan, and Department of Chemistry, National Taiwan University, Taipei 10617, Taiwan
}

Received August 11, 2007; E-mail: chungwu@cc.ee.ntu.edu.tw; kenwong@ntu.edu.tw

\begin{abstract}
White electroluminescent (EL) emission from single-layered solid-state light-emitting electrochemical cells (LECs) based on host-guest cationic iridium complexes has been successfully demonstrated. The devices show white EL spectra (Commission Internationale de l'Eclairage coordinates ranging from $(x, y)=(0.45,0.40)$ to $(0.35,0.39)$ at $2.9-3.3 \mathrm{~V}$ with high color rendering indices up to 80 . Peak external quantum efficiency and peak power efficiency of the white LEC reach $4 \%$ and $7.8 \mathrm{Im} / \mathrm{W}$, respectively. These results suggest that white LECs based on host-guest cationic transition metal complexes may be a promising alternative for solid-state lighting technologies.
\end{abstract}

\section{Introduction}

White organic light-emitting diodes (OLEDs) based on polymers and small-molecule materials have attracted much attention due to their potential applications in flat-panel displays and solid-state lighting. ${ }^{1}$ Compared with conventional white OLEDs, ${ }^{2}$ solid-state white light-emitting electrochemical cells $(\text { LECs })^{3}$ possess several promising advantages. LECs generally require only a single emissive layer, which can be easily processed from solutions, and can conveniently use air-stable electrodes. The emissive layer of LECs contains mobile ions, which can drift toward electrodes under an applied bias. The spatially separated ions induce doping (oxidation and reduction) of the emissive materials near the electrodes, that is, p-type doping near the anode and n-type doping near the cathode. ${ }^{3}$ The doped regions induce ohmic contacts with the electrodes and consequently facilitate the injection of both holes and electrons, which recombine at the junction between $\mathrm{p}$ - and n-type regions. As a result, a single-layered LEC device can be operated at very low voltages (close to $E_{\mathrm{g}} / e$, where $E_{\mathrm{g}}$ is the energy gap of the emissive material and $e$ is elementary charge) with balanced carrier injection, giving high power efficiencies. Furthermore, air-stable metals, for example, $\mathrm{Au}$ and $\mathrm{Ag}$, can be used since carrier injection in LECs is relatively insensitive to work functions of electrodes.

\footnotetext{
$\dagger$ Department of Electrical Engineering.

Department of Chemistry.

(1) (a) Kido, J.; Hongawa, K.; Okuyama, K.; Nagai, K. Appl. Phys. Lett. 1994, 64, 815. (b) Tokito, S.; Iijima, T.; Tsuzuki, T.; Sato, F. Appl. Phys. Lett. 2003, 83, 2459. (c) D'Andrade, B. W.; Forrest, S. R. Adv. Mater. 2004 16, 1585. (d) Gong, X.; Wang, S.; Moses, D.; Bazan, G. C.; Heeger, A. J. Adv. Mater. 2005, 17, 2053. (e) Huang, J.; Li, G.; Wu, E.; Xu, Q.; Yang, Y. Adv. Mater. 2006, 18, 114.

(2) (a) Tang, C. W.; VanSlyke, S. A. Appl. Phys. Lett. 1987, 51, 913. (b) Burroughes, J. H.; Bradley, D. D. C.; Brown, A. R.; Marks, R. N.; Mackay, K.; Friend, R. H.; Burns, P. L.; Holmes, A. B. Nature 1990, 347, 539.

(3) (a) Pei, Q.; Yu, G.; Zhang, C.; Yang, Y.; Heeger, A. J. Science 1995, 269 1086. (b) Pei, Q.; Yang, Y.; Yu, G.; Zhang, C.; Heeger, A. J. J. Am. Chem. Soc. 1996, 118, 3922. (c) Yang, Y.; Pei, Q. J. Appl. Phys. 1997, 81, 3294.
}

10.1021/ja076051e CCC: $\$ 40.75$ @ 2008 American Chemical Society
The only previous report of solid-state white LECs was based on phase-separated mixture of a polyfluorene derivative and poly(ethylene oxide) $(\mathrm{PEO})^{3 \mathrm{c}}$ However, the fluorescent nature of conjugated polymers would limit the eventual electroluminescence (EL) efficiency. Recently, cationic transition metal complexes have also been used in solid-state LECs,${ }^{4}$ which show two major advantages over conventional polymer LECs: (i) no ion-conducting material (e.g., PEO) is needed since these metal complexes are intrinsically ionic; (ii) higher EL efficiencies could be achieved due to the phosphorescent nature of the transition metal complexes. Inspired by previous works regard-

(4) (a) Lee, J. K.; Yoo, D. S.; Handy, E. S.; Rubner, M. F. Appl. Phys. Lett 1996, 69, 1686. (b) Lyons, C. H.; Abbas, E. D.; Lee, J. K.; Rubner, M. F. J. Am. Chem. Soc. 1998, 120, 12100. (c) Rudmann, H.; Rubner, M. F. J. Appl. Phys. 2001, 90, 4338. (d) Kalyuzhny, G.; Buda, M.; McNeill, J.; Barbara, P.; Bard, A. J. J. Am. Chem. Soc. 2003, 125, 6272. (e) Slinker, J. D.; Bernards, D.; Houston, P. L.; Abruña, H. D.; Bernhard, S.; Malliaras, G. G. Chem. Commun. 2003, 2392. (f) Rudmann, H.; Shimada, S.; Rubner, M. F. J. Appl. Phys. 2003, 94, 115. (g) Slinker, J. D.; Gorodetsky, A. A.; Lowry, M. S.; Wang, J.; Parker, S.; Rohl, R.; Bernhard, S.; Malliaras, G. G. J. Am. Chem. Soc. 2004, 126, 2763. (h) Slinker, J. D.; Malliaras, G. G.; Flores-Torres, S.; Abruña, H. D.; Chunwachirasiri, W.; Winokur, M. J. J. Appl. Phys. 2004, 95, 4381. (i) Hosseini, A. R.; Koh, C. Y.; Slinker, J. D. Flores-Torres, S.; Abruña, H. D.; Malliaras, G. G. Chem. Mater. 2005, 17 , 6114. (j) Slinker, J. D.; Koh, C. Y.; Malliaras, G. G.; Lowry, M. S.; Bernhard, S. Appl. Phys. Lett. 2005, 86, 173506. (k) Parker, S. T.; Slinker, J. D.; Lowry, M. S.; Cox, M. P.; Bernhard, S.; Malliaras, G. G. Chem. Mater. 2005, 17, 3187. (1) Lowry, M. S.; Goldsmith, J. I.; Slinker, J. D. Rohl, R.; Pascal, R. A., Jr.; Malliaras, G. G.; Bernhard, S. Chem. Mater 2005, 17, 5712. (m) Tamayo, A. B.; Garon, S.; Sajoto, T.; Djurovich, P. I.; Tsyba, I. M.; Bau, R.; Thompson, M. E. Inorg. Chem. 2005, 44, 8723 (n) Coppo, P.; Duati, M.; Kozhevnikov, V. N.; Hofstraat, J. W.; De Cola L. Angew. Chem., Int. Ed. 2005, 44, 1806. (o) Nazeeruddin, Md. K.; Wegh, R. T.; Zhou, Z.; Klein, C.; Wang, Q.; Angelis, F. De; Fantacci, S.; Grätzel, M. Inorg. Chem. 2006, 45, 9245. (p) Su, H. C.; Fang, F. C.; Hwu, T. Y.; Hsieh, H. H.; Chen, H. F.; Lee, G. H.; Peng, S. M.; Wong, K. T.; Wu, C. C. Adv. Funct. Mater. 2007, 17, 1019. (q) Su, H. C.; Wu, C. C.; Fang, F. C.; Wong, K. T. Appl. Phys. Lett. 2006, 89, 261118. (r) Soltzberg, L. J Slinker, J. D.; Flores-Torres, S.; Bernards, D. A.; Malliaras, G. G.; Abruña, H. D.; Kim, J.-S.; Friend, R. H.; Kaplan, M. D.; Goldberg, V. J. Am. Chem. Soc., 2006, 128, 7761. (s) Slinker, J. D.; Kim, J.-S.; Flores-Torres, S.; Delcamp, J. H.; Abruña, H. D.; Friend, R. H.; Malliaras, G. G. J. Mater. Chem., 2007, 17, 76. (t) Slinker, J. D.; Rivnay, J.; Moskowitz, J. S.; Parker, J. B.; Bernhard, S.; Abruña, H. D.; Malliaras, G. G. J. Mater. Chem. 2007, 17,2976 
ing energy transfer between ionic complexes reported by Malliaras $^{4 \mathrm{i}}$ and De Cola, ${ }^{4 \mathrm{n}}$ we had previously demonstrated highly efficient solid-state LEC devices by adopting host-guest cationic metal complexes. ${ }^{4 \mathrm{q}}$ Yet to our knowledge, there is no white LEC based on cationic transition metal complexes reported to date, despite their high potential.

Efficient white light emission may be most easily achieved by mixing two complementary colors, such as blue-green and red emission. Thus the development of efficient blue-green${ }^{41-o}$ and red-emitting ${ }^{4 a-i, m}$ cationic transition metal complexes is highly desired. In this paper, we report the synthesis and characterization of efficient blue-green- and red-emitting cationic iridium complexes and their successful application in white LECs with adopting the effective host-guest strategy. ${ }^{4 i, q}$ The white LECs show EL with Commission Internationale de l'Eclairage (CIE $)^{5}$ coordinates ranging from $(x, y)=(0.45,0.40)$ to $(0.35,0.39)$ at $2.9-3.3 \mathrm{~V}$, high color rendering indices $(\mathrm{CRI})^{6}$ up to 80 , external quantum efficiency (EQE) and power efficiency of up to $4 \%$ and $7.8 \mathrm{~lm} / \mathrm{W}$, respectively. These results demonstrate the high potential of white LECs based on hostguest cationic transition metal complexes for solid-state lighting.

\section{Experimental Section}

General Experiments. ${ }^{1} \mathrm{H}$ and ${ }^{13} \mathrm{C}$ NMR spectra of compounds were collected on a $400 \mathrm{MHz}$ spectrometer at room temperature. Photophysical characteristics of complexes in solutions were collected at room temperature by using $10^{-5} \mathrm{M}$ dichloromethane (DCM) solutions of all complexes, which were carefully purged with nitrogen prior to measurements. The neat, 1-butyl-3-methylimidazolium hexafluorophosphate $\left[\mathrm{BMIM}^{+}\left(\mathrm{PF}_{6}{ }^{-}\right)\right]$blended films $(\sim 100 \mathrm{~nm})$ for photoluminescence (PL) studies were spin-coated onto quartz substrates from acetonitrile $(\mathrm{MeCN})$ solutions. UV-visible absorption spectra were recorded on a spectrophotometer. PL spectra were measured with a cooled charge coupled device (CCD) coupled to a monochromator using the 325-nm line of the $\mathrm{He}-\mathrm{Cd}$ laser as the excitation. Photoluminescence quantum yields (PLQYs) for solution and thin-film samples were determined with a calibrated integrating sphere system. Excited-state lifetimes of samples were measured using the time-correlated singlephoton counting technique. ${ }^{4 p}$ Oxidation and reduction potentials of all complexes were determined by cyclic voltammetry (CV) at a scan rate of $100 \mathrm{mV} / \mathrm{s}$ in $\mathrm{MeCN}$ or DCM solutions $(1.0 \mathrm{mM})$. A glassy carbon electrode and a platinum wire were used as the working electrode and the counter electrode, respectively. All potentials were recorded versus the $\mathrm{Ag} / \mathrm{AgCl}$ (sat'd) reference electrode. Oxidation CV was performed using $0.1 \mathrm{M}$ tetra- $n$-butylammonium hexafluorophosphate $\left(\mathrm{TBAPF}_{6}\right)$ in $\mathrm{MeCN}$ (for 1 and 2), 0.1 M TBAPF 6 in DCM (for 3), and $0.1 \mathrm{M}$ tetra- $n$-butylammonium perchlorate (TBAP) in $\mathrm{MeCN}$ (for 4) as the supporting electrolyte. For reduction CV, 0.1 M TBAP in MeCN (for all complexes) was used as the supporting electrolyte.

Fabrication and Characterization of LEC Devices. LEC devices were fabricated by spin-coating the mixed solutions of complexes 3 and 4 and $\mathrm{BMIM}^{+}\left(\mathrm{PF}_{6}{ }^{-}\right)(80.5,0.4$, and $19.1 \mathrm{wt} . \%$, respectively) on indium-tin-oxide coated glass substrates to form a $\sim 100$-nm thick film, followed by thermal evaporation of a $150-\mathrm{nm} \mathrm{Ag}$ top contact (cathode). $\mathrm{BMIM}^{+}\left(\mathrm{PF}_{6}{ }^{-}\right)$was added to provide additional anions, which shortened the device response time. ${ }^{4 \mathrm{k}}$ The electrical and emission characteristics of LECs were measured under constant bias voltages. The device was driven with a source-measurement unit (SMU) and the emission intensity was measured using a Si photodiode calibrated with Photo Research PR650. EL spectra of devices were measured by a calibrated spectrometer with a CCD array detector.

(5) Colorimety; Commission Internationale de l'Eclairage (CIE): Paris, 1986 (6) Method of Measuring and Specifying Colour Rendering Properties of Light Sources; Commission Internationale de l'Eclairage (CIE): Paris, 1974.
General Procedures for Synthesis. The compound 1- $\left(2^{\prime}, 4^{\prime}-\right.$ difluorophenyl)pyrazole was prepared following a literature procedure. ${ }^{7} 4,5$ Diaza-9,9'-spirobifluorene (dasb) ${ }^{8 a}$ and 9,9-bis(4-methoxyphenyl)-4,5diazafluorene (bmpdaf) ${ }^{8 b}$ were prepared according to literature procedures. All experiments involving $\operatorname{IrCl}_{3} \cdot \mathrm{H}_{2} \mathrm{O}$ or any other $\operatorname{Ir}(\mathrm{III})$ species were carried out in an inert atmosphere. Cyclometalated $\operatorname{Ir}(\mathrm{III})$ dichlorobridged dimers of the general formula $\left(\mathrm{C}^{\wedge} \mathrm{N}\right)_{2} \operatorname{Ir}(\mu-\mathrm{Cl})_{2} \operatorname{Ir}\left(\mathrm{C}^{\wedge} \mathrm{N}\right)_{2}$ (where $\mathrm{C}^{\wedge} \mathrm{N}$ represents a cyclometalating ligand) were synthesized by a literature procedure. ${ }^{9}$

Synthesis of 9,9-Diethyl-4,5-diazafluorene (dedaf). A mixture of 4,5-diazafluorene ${ }^{8 c}(600 \mathrm{mg}, 3.57 \mathrm{mmol})$, ethylbromide $(0.8 \mathrm{~mL}, 10.7$ $\mathrm{mmol})$, and potassium tert-butoxide $(1.2 \mathrm{~g}, 10.7 \mathrm{mmol})$ was dissolved in $15 \mathrm{~mL}$ of THF and stirred for $3 \mathrm{~h}$ at room temperature. The solvent was evaporated, and the reaction mixture was extracted with dichloromethane and dried with $\mathrm{MgSO}_{4}$ to afford pure product (733 mg, $92 \%$ yield) as a sticky dark fluid. ${ }^{1} \mathrm{H}$ NMR $\left(\mathrm{CDCl}_{3}, 400 \mathrm{MHz}\right) \delta 8.66(\mathrm{dd}$, $J=4.8,1.6 \mathrm{~Hz}, 2 \mathrm{H}), 7.72(\mathrm{dd}, J=8.0,1.6 \mathrm{~Hz}, 2 \mathrm{H}), 7.31(\mathrm{dd}, J=$ $8.0,4.8,4 \mathrm{H}), 2.07$ (q, $J=7.2,4 \mathrm{H}), 0.38(\mathrm{t}, J=7.2,6 \mathrm{H}) ;{ }^{13} \mathrm{C}$ NMR $\left(\mathrm{CDCl}_{3}, 100 \mathrm{MHz}\right) \delta 149.3,131.1,123.2,117.8,115.2,63.8,28.4$, 8.6; $\mathrm{MS}\left(\mathrm{m} / \mathrm{z}, \mathrm{ESI}^{+}\right) 62$ (35), 195 (100), 224 (20); HRMS ( $\left.\mathrm{m} / \mathrm{z}, \mathrm{FAB}^{+}\right)$ calcd for $\mathrm{C}_{15} \mathrm{H}_{16} \mathrm{~N}_{2}$ 224.1313, found 224.1316.

Synthesis of $\left[\operatorname{Ir}(\mathbf{d f p p z})_{2}(\mathbf{d a s b})\right]^{+}\left(\mathbf{P F}_{6}{ }^{-}\right)(\mathbf{1})$. Bis- $(\mu)$-chlorotetrakis(1-(4,6-difluorophenyl)-pyrazolato- $\left.\mathrm{C}^{2}, \mathrm{~N}\right)$ diiridium(III) $(1.17 \mathrm{mg}, 1.0$ $\mathrm{mmol})$ and 4,5-diaza-9,9'-spirobifluorene ${ }^{8 \mathrm{a}}(668 \mathrm{mg}, 2.1 \mathrm{mmol})$ were dissolved in 1,2-ethanediol $(70 \mathrm{~mL})$ under $\mathrm{Ar}$, and the solution was kept at $150^{\circ} \mathrm{C}$ for $16 \mathrm{~h}$. The solution was cooled to room temperature, and an aqueous solution of $\mathrm{NH}_{4} \mathrm{PF}_{6}(2.5 \mathrm{mg}$ in $20 \mathrm{~mL}$ of deionized water) was added to yield a yellow suspension. The solid was then filtered and dried in an oven $\left(80^{\circ} \mathrm{C}\right)$ for $12 \mathrm{~h}$. The crude product was purified by column chromatography on silica gel $\left(\mathrm{CH}_{2} \mathrm{Cl}_{2} / \mathrm{MeCN}=\right.$ 10/1) to give compound $1(1.5 \mathrm{~g}, 87 \%)$ as a yellow solid. ${ }^{1} \mathrm{H}$ NMR $\left(\mathrm{CDCl}_{3}, 400 \mathrm{MHz}\right) \delta 8.40(\mathrm{~d}, J=2.4 \mathrm{~Hz}, 2 \mathrm{H}), 7.89-7.85(\mathrm{~m}, 4 \mathrm{H})$, $7.46(\mathrm{t}, J=7.8 \mathrm{~Hz}, 2 \mathrm{H}), 7.41(\mathrm{~d}, J=2.4,4 \mathrm{H}), 7.37(\mathrm{~d}, J=2.4,2 \mathrm{H})$, $7.22(\mathrm{~d}, J=8.0,2 \mathrm{H}), 6.78-6.76(\mathrm{~m}, 4 \mathrm{H}), 6.69(\mathrm{td}, J=10,2.6 \mathrm{~Hz}$, $2 \mathrm{H}), 5.85(\mathrm{dd}, J=8.0,2.4 \mathrm{~Hz}, 2 \mathrm{H}) ;{ }^{13} \mathrm{C} \mathrm{NMR}\left(\mathrm{CDCl}_{3}, 100 \mathrm{MHz}\right) \delta$ $162.1,149.8,148.6,143.6,141.7,140.9,139.0,135.2,131.7,131.5$, 129.6, 128.9, 128.1, 123.9, 120.8, 115.5, 115.3, 109.7, 99.9, 99.7, 67.1; $\operatorname{MS}\left(\mathrm{m} / z, \mathrm{ESI}^{+}\right) 381(15), 869(100)$; HRMS $\left(\mathrm{m} / z, \mathrm{FAB}^{+}\right)$calcd for $\mathrm{C}_{41} \mathrm{H}_{24} \mathrm{~F}_{4} \operatorname{IrN}_{6}$ 869.1628, found 869.1644.

Synthesis of $\left[\operatorname{Ir}(\mathbf{d f p p z})_{2}(\mathbf{b m p d a f})\right]^{+}\left(\mathbf{P F}_{6}{ }^{-}\right)(\mathbf{2})$. Bis- $(\mu)$-chlorotetrakis(1-(4,6-difluorophenyl)-pyrazolato- $\left.\mathrm{C}^{2}, \mathrm{~N}\right)$ diiridium(III) (350 mg, 0.3 $\mathrm{mmol}$ ) and 9,9-bis(4-methoxyphenyl)-4,5-diazafluorene ${ }^{8 \mathrm{~b}}$ (239 mg, 0.63 mmol) were dissolved in 1,2-ethanediol $(25 \mathrm{~mL})$ under Ar, and the solution was kept at $150{ }^{\circ} \mathrm{C}$ for $16 \mathrm{~h}$. The solution was cooled to room temperature, and an aqueous solution of $\mathrm{NH}_{4} \mathrm{PF}_{6}(750 \mathrm{mg}$ in $7.5 \mathrm{~mL}$ of deionized water) was added to yield a yellow suspension. The solid was then filtered and dried in an oven $\left(80{ }^{\circ} \mathrm{C}\right)$ for $12 \mathrm{~h}$. The crude product was purified by column chromatography on silica gel $\left(\mathrm{CH}_{2-}\right.$ $\left.\mathrm{Cl}_{2} / \mathrm{MeCN}=10 / 1\right)$ to give compound $2(525 \mathrm{mg}, 82 \%)$ as a yellow solid. ${ }^{1} \mathrm{H} \mathrm{NMR}\left(\mathrm{CDCl}_{3}, 400 \mathrm{MHz}\right) \delta 8.34(\mathrm{~d}, J=2.4 \mathrm{~Hz}, 2 \mathrm{H}), 8.05$ $(\mathrm{d}, J=7.2 \mathrm{~Hz}, 2 \mathrm{H}), 7.85(\mathrm{~d}, J=4.4 \mathrm{~Hz}, 2 \mathrm{H}), 7.58(\mathrm{dd}, J=8.0,5.2$ $\mathrm{Hz}, 2 \mathrm{H}), 7.16(\mathrm{~d}, J=2.4 \mathrm{~Hz}, 2 \mathrm{H}), 7.09(\mathrm{dd}, J=6.8,2.0 \mathrm{~Hz}, 4 \mathrm{H})$, $6.85(\mathrm{dd}, J=6.8,2.0 \mathrm{~Hz}, 4 \mathrm{H}), 6.70-6.64(\mathrm{~m}, 4 \mathrm{H}), 5.80(\mathrm{dd}, J=8.0$, $2.4 \mathrm{~Hz}, 2 \mathrm{H}), 3.77(\mathrm{~s}, 6 \mathrm{H}) ;{ }^{13} \mathrm{C} \mathrm{NMR}\left(\mathrm{CDCl}_{3}, 100 \mathrm{MHz}\right) \delta 160.4,159.5$, $148.3,145.7,142.6,138.8,136.9,131.9,131.6,131.4,130.3,128.2$, $127.9,117.1,115.4,115.2,114.7,109.5,93.6,86.6,67.7,55.5 ; \mathrm{MS}$ $\left(\mathrm{m} / \mathrm{z}, \mathrm{ESI}^{+}\right) 261(10), 381(20), 931(100) ; \operatorname{HRMS}\left(\mathrm{m} / \mathrm{z}, \mathrm{FAB}^{+}\right)$calcd for $\mathrm{C}_{43} \mathrm{H}_{30} \mathrm{~F}_{4} \mathrm{IrN}_{6} \mathrm{O}_{2}$ 931.1996, found 931.2019.

(7) (a) Finar, I. L.; Rackham, D. M. J. Chem. Soc. B 1968, 211. (b) Finar, I. L.; Godfrey, K. E. J. Chem. Soc. 1954, 2293.

(8) (a) Wong, K. T.; Chen, R. T.; Fang, F. C.; Wu, C. C.; Lin, Y. T. Org. Lett 2005, 7, 1979. (b) Ono, K.; Yanase, T.; Ohkita, M.; Saito, K.; Matsushita, Y.; Naka, S.; Okada, H.; Onnagawa, H. Chem. Lett. 2004, 33, 276. (c) Thummel, R. P.; Lefoulon, F.; Mahadevan, R. J. Org. Chem. 1985, 50, 3824.

(9) (a) Sprouse, S.; King, K. A.; Spellane, P. J.; Watts, R. J. J. Am. Chem. Soc. 1984, 106, 6647. (b) Lohse, O.; Thevenin, P.; Waldvogel, E. Synlett 1999, 1, 45. (c) Nonoyama, M. Bull. Chem. Soc. Jpn. 1974, 47, 767. 
Scheme 1. Synthetic Pathways and Structures of Complexes 1, 2, 3, and 4
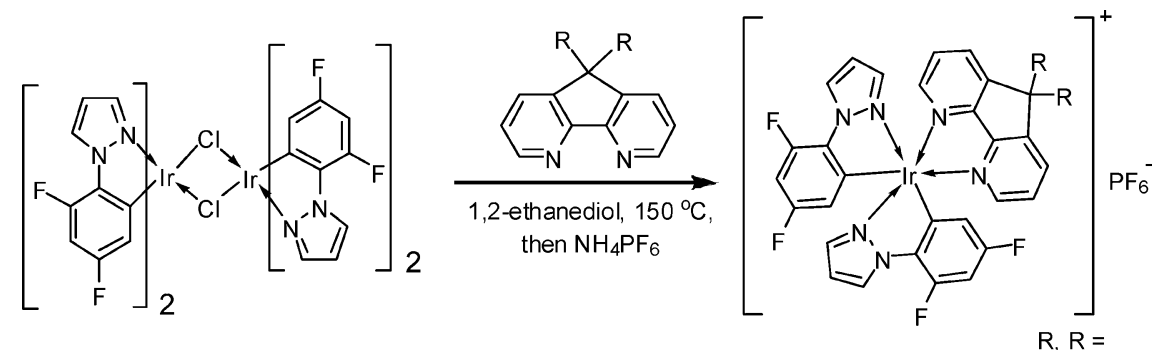

1: $\left[\operatorname{lr}(\mathrm{dfppz})_{2}(\mathrm{dasb})\right]^{+}\left(\mathrm{PF}_{6}^{-}\right)$

$R, R=$

2: $\left[\operatorname{lr}(\mathrm{dfppz})_{2}(\text { bmpdaf })\right]^{+}\left(\mathrm{PF}_{6}^{-}\right)$

3: $\left[\operatorname{Ir}(\mathrm{dfppz})_{2}(\mathrm{dedaf})\right]^{+}\left(\mathrm{PF}_{6}^{-}\right)$

2,2'-biphenyl

4- $\mathrm{CH}_{3} \mathrm{OPh}, 4-\mathrm{CH}_{3} \mathrm{OPh}$

$\mathrm{C}_{2} \mathrm{H}_{5}, \mathrm{C}_{2} \mathrm{H}_{5}$
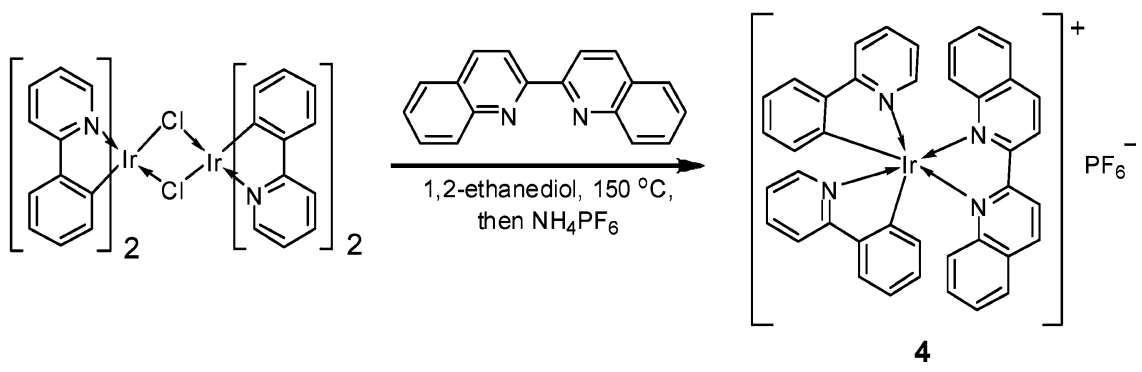

Synthesis of $\left[\operatorname{Ir}(\mathbf{d f p p z})_{\mathbf{2}}(\mathbf{d e d a f})\right]^{+}\left(\mathbf{P F}_{\mathbf{6}}{ }^{-}\right)(\mathbf{3})$. Bis- $(\mu)$-chlorotetrakis(1-(4,6-difluorophenyl)-pyrazolato- $\left.\mathrm{C}^{2}, \mathrm{~N}\right)$ diiridium(III) (373 mg, 0.32 $\mathrm{mmol}$ ) and 9,9-diethyl-4,5-diazafluorene $(150 \mathrm{mg}, 0.67 \mathrm{mmol})$ were dissolved in 1,2-ethanediol ( $25 \mathrm{~mL})$ under Ar, and the solution was kept at $150{ }^{\circ} \mathrm{C}$ for $16 \mathrm{~h}$. The solution was cooled to room temperature, and an aqueous solution of $\mathrm{NH}_{4} \mathrm{PF}_{6}(750 \mathrm{mg}$ in $7.5 \mathrm{~mL}$ of deionized water) was added to yield a yellow suspension. The solid was then filtered and dried in an oven $\left(80{ }^{\circ} \mathrm{C}\right)$ for $12 \mathrm{~h}$. The crude product was purified by column chromatography on silica gel $\left(\mathrm{CH}_{2} \mathrm{Cl}_{2} / \mathrm{MeCN}=\right.$ 10/1) to give compound 3 (480 mg, 82\%) as a yellow solid. ${ }^{1} \mathrm{H}$ NMR $\left(\mathrm{CDCl}_{3}, 400 \mathrm{MHz}\right) \delta 8.29(\mathrm{~d}, J=8.0 \mathrm{~Hz}, 2 \mathrm{H}), 8.08(\mathrm{dd}, J=8.0,0.8$ $\mathrm{Hz}, 2 \mathrm{H}), 7.80(\mathrm{t}, J=8.0 \mathrm{~Hz}, 2 \mathrm{H}), 7.72(\mathrm{dd}, J=5.2,0.8 \mathrm{~Hz}, 2 \mathrm{H})$, $7.64-7.58(\mathrm{~m}, 2 \mathrm{H}), 7.09$ (t, $J=6.4 \mathrm{~Hz}, 2 \mathrm{H}), 6.57(\mathrm{td}, J=10.6,2.4$, $2 \mathrm{H}), 5.77(\mathrm{dd}, J=8.0,2.4 \mathrm{~Hz}, 2 \mathrm{H}), 2.21(\mathrm{q}, J=7.2 \mathrm{~Hz}, 4 \mathrm{H}), 0.53(\mathrm{t}$, $J=7.2 \mathrm{~Hz}, 6 \mathrm{H}) ;{ }^{13} \mathrm{C} \mathrm{NMR}\left(\mathrm{CDCl}_{3}, 100 \mathrm{MHz}\right) \delta 161.3,147.7,147.3$, 144.5, 139.1, 138.4, 135.3, 131.6, 131.4, 127.9, 127.4, 123.6, 115.5, 109.3, 99.8, 99.6, 61.1, 29.9, 9.0; MS ( $\left.\mathrm{m} / z, \mathrm{ESI}^{+}\right) 551$ (15), 775 (100); HRMS $\left(\mathrm{m} / z, \mathrm{FAB}^{+}\right)$calcd for $\mathrm{C}_{33} \mathrm{H}_{26} \mathrm{~F}_{4} \mathrm{IrN}_{6} 775.1784$, found 775.1780 .

Synthesis of $\left[\operatorname{Ir}(\mathbf{p p y})_{2}(\mathbf{b i q})\right]^{+}\left(\mathbf{P F}_{\mathbf{6}}{ }^{-}\right) \mathbf{( 4 )}$. Bis- $(\mu)$-chlorotetrakis(2phenylpyridinato- $\left.\mathrm{C}^{2}, \mathrm{~N}\right)$ diiridium(III) $(398 \mathrm{mg}, 0.37 \mathrm{mmol})$ and $2,2^{\prime}-$ biquinoline (200 mg, $0.78 \mathrm{mmol}$ ) were dissolved in 1,2-ethanediol (30 $\mathrm{mL}$ ) under Ar, and the solution was kept at $150{ }^{\circ} \mathrm{C}$ for $16 \mathrm{~h}$. The solution was cooled to room temperature, and an aqueous solution of $\mathrm{NH}_{4} \mathrm{PF}_{6}$ ( $800 \mathrm{mg}$ in $8 \mathrm{~mL}$ of deionized water) was added to yield a red suspension. The solid was then filtered and dried in an oven $\left(80^{\circ} \mathrm{C}\right)$ for $12 \mathrm{~h}$. The crude product was purified by column chromatography on silica gel $\left(\mathrm{CH}_{2} \mathrm{Cl}_{2} / \mathrm{MeCN}=10 / 1\right)$ to give compound $4(658 \mathrm{mg}$, 98\%) as a red solid. ${ }^{1} \mathrm{H}$ NMR $\left(\mathrm{CDCl}_{3}, 400 \mathrm{MHz}\right) \delta 8.95(\mathrm{~d}, J=9.2$ $\mathrm{Hz}, 2 \mathrm{H}), 8.88(\mathrm{~d}, J=9.2 \mathrm{~Hz}, 2 \mathrm{H}), 8.13(\mathrm{~d}, J=8.0 \mathrm{~Hz}, 2 \mathrm{H}), 8.08(\mathrm{~d}$, $J=8.0 \mathrm{~Hz}, 2 \mathrm{H}), 7.87-7.83(\mathrm{~m}, 6 \mathrm{H}), 7.77(\mathrm{dd}, J=8.0,1.2 \mathrm{~Hz}, 2 \mathrm{H})$, 7.57 (t, $J=8.0 \mathrm{~Hz}, 2 \mathrm{H}), 7.14(\mathrm{t}, J=8.0 \mathrm{~Hz}, 2 \mathrm{H}), 7.04(\mathrm{q}, J=8.0 \mathrm{~Hz}$, $2 \mathrm{H}), 6.95(\mathrm{t}, J=8.0 \mathrm{~Hz}, 2 \mathrm{H}), 6.85(\mathrm{t}, J=8.0 \mathrm{~Hz}, 2 \mathrm{H}), 6.12(\mathrm{~d}, J=$ $8.0 \mathrm{~Hz}, 2 \mathrm{H}) ;{ }^{13} \mathrm{C} \mathrm{NMR}\left(\mathrm{CDCl}_{3}, 100 \mathrm{MHz}\right) \delta 159.5,149.7,148.0,147.7$, $142.4,141.5,137.8,134.1,131.0,130.4,129.7,128.6,127.8,127.1$, 124.6, 124.1, 123.0, 122.5, 122.3, 119.3; MS $\left(\mathrm{m} / \mathrm{z}, \mathrm{ESI}^{+}\right) 501$ (100), 757 (70); HRMS $\left(\mathrm{m} / \mathrm{z}, \mathrm{ESI}^{+}\right)$calcd for $\mathrm{C}_{40} \mathrm{H}_{28} \mathrm{IrN}_{4} 757.1943$, found 757.1946 .

\section{Results and Discussion}

To date, reports on blue (or blue-green)-emitting cationic transition metal complexes with stable redox properties, which are essential for LEC applications, are limited. ${ }^{41-0}$ Among them, the complex $\left[\operatorname{Ir}(\mathrm{dfppz})_{2}(\mathrm{dtb}-\mathrm{bpy})\right]^{+}\left(\mathrm{PF}_{6}\right)^{-}$(where dfppz is $1-(2,4-$ difluorophenyl)pyrazole and dtb-bpy is [4,4'-di(tert-butyl)-2,2'bipyridine]) exhibits the shortest neat-film emission wavelength of $487 \mathrm{~nm} .{ }^{4 \mathrm{~m}}$ The large energy gap $(3.08 \mathrm{eV})$ of $\left[\operatorname{Ir}(\mathrm{dfppz})_{2}\right.$ $(\mathrm{dtb}-\mathrm{bpy})]^{+}\left(\mathrm{PF}_{6}\right)^{-}$can be reasonably attributed to the tailormade dfppz ligand, which stabilizes the highest occupied molecular orbital (HOMO) and destabilizes the lowest unoccupied molecular orbital (LUMO) in a good manner. ${ }^{4 \mathrm{~m}}$ A small bathochromic shift (only $3 \mathrm{~nm}$ ) between neat-film emission and solution emission of this complex is possibly associated with the reduced intermolecular interactions provided by sterically bulky di-tert-butyl groups of the bipyridine ligand. To increase rigidity of the molecular structure, which could potentially result in higher photoluminescence quantum yields (PLQY), we introduced 4,5-diazafluorene (daf) as an ancillary ligand onto the dfppz-based cationic iridium complex. However, the synthesis of cationic iridium complexes using C9-unsubstituted daf was unsuccessful. Therefore, daf derivatives with aromatic or alkyl substitutions on $\mathrm{C} 9$ of daf, such as 4,5-diaza-9,9'spirobifluorene (dasb), 9,9-bis(4-methoxyphenyl)-4,5-diazafluorene (bmpdaf), and 9,9-diethyl-4,5-diazafluorene (dedaf), were used instead. The synthesis of cationic iridium complexes $\left[\operatorname{Ir}(\mathrm{dfppz})_{2}(\mathrm{dasb})\right]^{+}\left(\mathrm{PF}_{6}{ }^{-}\right)(\mathbf{1}),\left[\operatorname{Ir}(\mathrm{dfppz})_{2}(\mathrm{bmpdaf})\right]^{+}\left(\mathrm{PF}_{6}{ }^{-}\right)(\mathbf{2})$, and $\left[\operatorname{Ir}(\mathrm{dfppz})_{2}(\mathrm{dedaf})\right]^{+}\left(\mathrm{PF}_{6}{ }^{-}\right)(\mathbf{3})$ is shown in Scheme 1 . The reactions of daf derivatives with cyclometalated $\mathrm{Ir}(\mathrm{III})$ dichlorobridged dimers followed by subsequent ion exchanges to $\mathrm{PF}_{6}{ }^{-}$ afforded the desired cationic complexes with excellent yields $(>80 \%)$.

For the red-emitting complex, $\left[\operatorname{Ir}(\mathrm{ppz})_{2}(\mathrm{biq})\right]^{+}\left(\mathrm{PF}_{6}{ }^{-}\right)$(where ppz is 1-phenylpyrazole and biq is $2,2^{\prime}$-biquinoline) was previously reported to show neat-film emission around 622 $\mathrm{nm} .{ }^{4 \mathrm{~m}}$ To achieve white emission, the red-emitting guest is to be doped into the blue (or blue-green)-emitting host at a low concentration (generally $<1$ wt $\%$ ) to induce partial energy transfer. However, at such low concentrations, the emission usually shows significant blue-shift to orange or even yellow 
Scheme 2. The Crystal Structures of Complexes 3 (top) and 4 $(\text { bottom })^{a}$
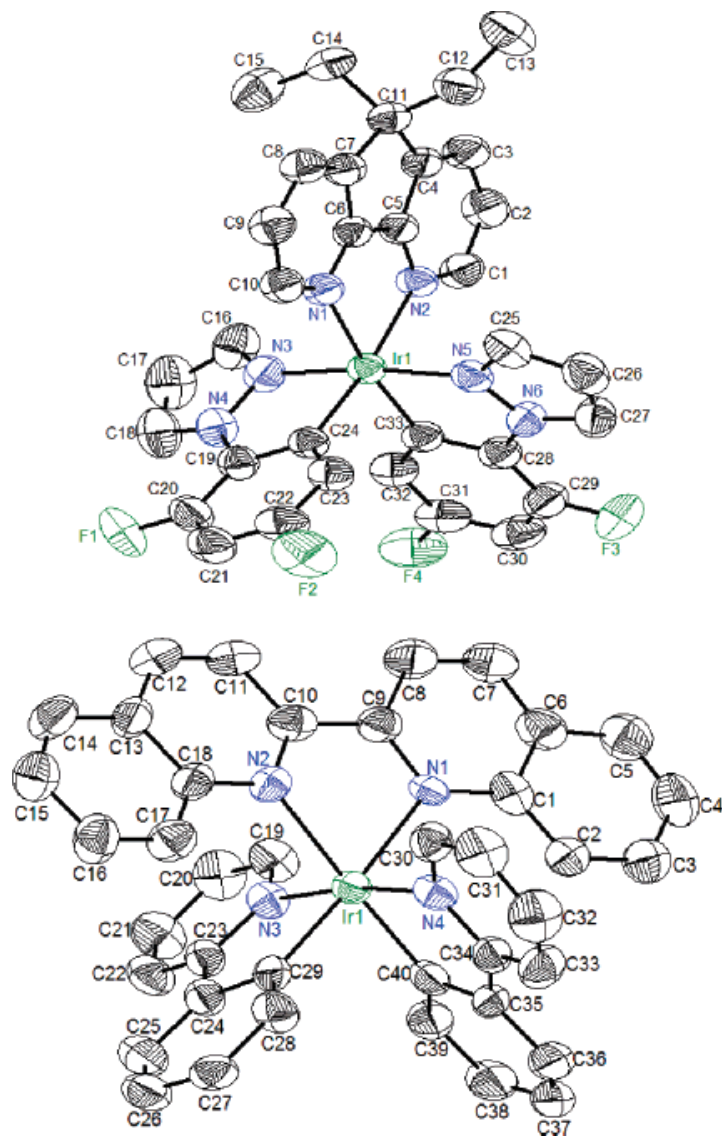

${ }^{a}$ Counter anion $\mathrm{PF}_{6}{ }^{-}$is omitted for clarity. Thermal ellipsoids are drawn at the $50 \%$ probability.

due to reduced intermolecular interactions. Therefore, a red emitter with an even lower energy gap is desired to ensure red emission even at low doping concentrations. To achieve this, $\left[\operatorname{Ir}(\mathrm{ppz})_{2}(\mathrm{biq})\right]^{+}\left(\mathrm{PF}_{6}{ }^{-}\right)$was modified by replacing ppz with 2-phenylpyridine (ppy), which exhibits lower triplet energy than $\mathrm{ppz},{ }^{4 \mathrm{~m}}$ to form $\left[\operatorname{Ir}(\mathrm{ppy})_{2}(\mathrm{biq})\right]^{+}\left(\mathrm{PF}_{6}{ }^{-}\right)$(4) (Scheme 1). Crystals of complex $\mathbf{3}$ and $\mathbf{4}$ suitable for the $\mathrm{X}$-ray diffraction analysis were obtained by slow evaporation of the solvent from the acetone/pentane cosolvent. The structures of complexes $\mathbf{3}$ and $\mathbf{4}$ are depicted in Scheme 2, and the crystal data are summarized in Table 1. The molecular structures shown in Scheme 2, complex 3 with two cyclometalated 1-(2,4-difluorophenyl)pyrazole $\left(\mathrm{C}^{\wedge} \mathrm{N}\right)$ ligands and one 9,9'-diethyl-4,5-diazafluorene $\left(\mathrm{N}^{\wedge} \mathrm{N}\right)$ ligand and complex 4 with cyclometalated 2-phenylpyridine $\left(\mathrm{C}^{\wedge} \mathrm{N}\right)$ ligands and one 2,2'-biquinoline $\left(\mathrm{N}^{\wedge} \mathrm{N}\right)$ ligand, exhibit a distorted octahedral geometry around the $\mathrm{Ir}$ center as indicated by the small bite angles of $\mathrm{C}(33)-\operatorname{Ir}(1)-\mathrm{N}(5)$ [80.39$\left.(15)^{\circ}\right], \mathrm{C}(24)-\operatorname{Ir}(1)-\mathrm{N}(3)\left[79.88(15)^{\circ}\right]$, and $\mathrm{N}(1)-\operatorname{Ir}(1)-\mathrm{N}(2)$ $\left[80.66(12)^{\circ}\right]$ and twisted bond angles of $\mathrm{N}(5)-\operatorname{Ir}(1)-\mathrm{N}(3)$ $\left[171.81(13)^{\circ}\right], \mathrm{C}(33)-\operatorname{Ir}(1)-\mathrm{N}(1)\left[171.91(13)^{\circ}\right]$, and $\mathrm{C}(24)-$ $\operatorname{Ir}(1)-\mathrm{N}(2)\left[173.59(13)^{\circ}\right]$ in complex 3 and small bite angles of $\mathrm{C}(40)-\operatorname{Ir}(1)-\mathrm{N}(4)\left[80.0(3)^{\circ}\right], \mathrm{C}(29)-\operatorname{Ir}(1)-\mathrm{N}(3)\left[80.7(4)^{\circ}\right]$, and $\mathrm{N}(1)-\operatorname{Ir}(1)-\mathrm{N}(2)\left[75.6(3)^{\circ}\right]$ and twisted bond angles of $\mathrm{N}(4)-\operatorname{Ir}(1)-\mathrm{N}(3)\left[171.2(3)^{\circ}\right], \mathrm{C}(40)-\operatorname{Ir}(1)-\mathrm{N}(2)\left[177.6(3)^{\circ}\right]$, and $\mathrm{C}(29)-\operatorname{Ir}(1)-\mathrm{N}(1)\left[174.0(4)^{\circ}\right]$ in complex 4 . The $\operatorname{Ir}-\mathrm{N}$ bond distances between the Ir center and 9,9'-diethyl-4,5diazafluorene and 2,2'-biquinoline are estimated to be $\operatorname{Ir}(1)-$ $\mathrm{N}(1)[2.177(3) \AA], \operatorname{Ir}(1)-\mathrm{N}(2) \quad[2.189(3) \AA]$ and $\operatorname{Ir}(1)-\mathrm{N}(1)$
Table 1. The Crystal Data of Complexes 3 and 4

\begin{tabular}{lll}
\hline & \multicolumn{1}{c}{3} & \multicolumn{1}{c}{4} \\
\hline empirical formula & $\mathrm{C}_{36} \mathrm{H}_{32} \mathrm{~F}_{10} \mathrm{IrN}_{6} \mathrm{OP}$ & $\mathrm{C}_{40} \mathrm{H}_{28} \mathrm{~F}_{6} \mathrm{IrN}_{4} \mathrm{P}$ \\
formula weight & 977.85 & 901.83 \\
crystal dimensions $\left(\mathrm{mm}^{3}\right)$ & $0.15 \times 0.10 \times 0.05$ & $0.20 \times 0.15 \times 0.10$ \\
crystal system & triclinic & monoclinic \\
space group & $P \overline{1}$ & $C c$ \\
$a(\AA)$ & $10.04400(10)$ & $13.9100(2)$ \\
$b(\AA)$ & $14.09140(10)$ & $13.0166(2)$ \\
$c(\AA)$ & $14.4291(2)$ & $19.9665(4)$ \\
$\alpha(\mathrm{deg})$ & $67.0550(10)$ & 90 \\
$\beta(\mathrm{deg})$ & $87.2080(10)$ & $105.8720(10)$ \\
$\gamma(\mathrm{deg})$ & $84.6510(10)$ & 90 \\
cell volume $(\AA)^{3}$ & $1872.27(3)$ & $3440.70(10)$ \\
$Z$ & 2 & 4 \\
density (calcd) $\left(\mathrm{g} / \mathrm{cm}^{3}\right)$ & 1.735 & 1.741 \\
$F(000)$ & 960 & 1768 \\
temp $(\mathrm{K})$ & $295(2)$ & $295(2)$ \\
wavelength $(\AA)$ & 0.71073 & 0.71073 \\
no. of reflns collected & 15981 & 12983 \\
no. of indep reflns $\left(R_{\text {int }}\right)$ & $8532(0.0282)$ & $7447(0.0467)$ \\
$\mathrm{R}(F)$, wR2 $[I>2 \sigma(I)]$ & $0.0339,0.0893$ & $0.0417,0.0989$ \\
$\mathrm{R}(F)$, wR2 (all data) & $0.0388,0.0955$ & $0.0531,0.1162$ \\
& & \\
\hline
\end{tabular}

[2.235(10) $\mathrm{A}], \operatorname{Ir}(1)-\mathrm{N}(2)$ [2.219(7) $\mathrm{A}]$, respectively, which are significantly longer than those between the Ir center and 1-(2,4difluorophenyl)pyrazole $[\operatorname{Ir}(1)-\mathrm{N}(3)=2.020(4) \AA, \operatorname{Ir}(1)-\mathrm{N}(5)$ $=2.020(4) \AA]$ or 2-phenylpyridine $[\operatorname{Ir}(1)-\mathrm{N}(3)=2.058(7) \AA$, $\operatorname{Ir}(1)-\mathrm{N}(4)=2.050(7) \AA]$. This can be possibly attributed to the anionic nature of the cyclometalated 1-(2,4-difluorophenyl)pyrazole and 2-phenylpyridine ligands, which have a stronger interaction with the cationic $\operatorname{Ir}(\mathrm{III})$ ion.

The emission properties of complexes $\mathbf{1 - 4}$ in solutions (DCM, $10^{-5} \mathrm{M}$ ) or in thin films are summarized in Table 2. For reducing the response time of LECs, in this work the ionic liquid $\mathrm{BMIM}^{+}\left(\mathrm{PF}_{6}{ }^{-}\right)$was introduced in the emissive layer to provide additional mobile anions. ${ }^{4 \mathrm{k}, \mathrm{p}, \mathrm{q}}$ Thus, emission properties of $\mathbf{1}-\mathbf{3}$ in films in the presence of $\mathrm{BMIM}^{+}\left(\mathrm{PF}_{6}{ }^{-}\right)(19 \mathrm{wt} \%)$ were also examined. In general, complexes $\mathbf{1}-\mathbf{3}$ show PL peaks at $491-499 \mathrm{~nm}$ and rather high PLQYs of $0.46-0.66$ in solutions. The high PLQYs of these complexes indicate that the rigidity of the daf ligand is beneficial for reducing nonradiative (e.g., vibration) deactivation processes. Among the three blue-green complexes $\mathbf{1 - 3}, \mathbf{3}$ exhibits shortest emission wavelengths of $488-491 \mathrm{~nm}$ and highest PLQYs of $0.28-0.30$ in thin films (neat or dispersed with $\mathrm{BMIM}^{+}\left(\mathrm{PF}_{6}{ }^{-}\right)$) and thus was chosen as the host material for white LEC studies. The absorption and PL spectra of blue-green complex $\mathbf{3}$ and redemitting complex 4 in solutions and in neat films $(\sim 100 \mathrm{~nm})$ are explicitly shown in Figure 1. Both complexes show intense absorption bands (with the extinction $\epsilon>3 \times 10^{4} \mathrm{M}^{-1} \mathrm{~cm}^{-1}$ ) in the ultraviolet region ranging between 200 and $\sim 300 \mathrm{~nm}$. These bands are associated with the ligand-centered (LC) transitions. ${ }^{41, \mathrm{~m}}$ These LC bands are accompanied by weaker and broad bands extending from $\sim 300 \mathrm{~nm}$ to the visible region, which are related to the metal-to-ligand-charge-transfer (MLCT) transitions (both allowed and spin-forbidden ones) mediated by the strong spin-orbit coupling of the $\operatorname{Ir}(\mathrm{III})$ center. ${ }^{41, \mathrm{~m}}$ Interestingly, complex $\mathbf{3}$ with the alkyl substitution on daf exhibits nearly the same emission wavelengths in solutions and in solid films. Complex 4 exhibits more saturated red emission (with PL peaks of 656 and $672 \mathrm{~nm}$ in solutions and in neat films, respectively) compared with that of $\left[\operatorname{Ir}(\mathrm{ppz})_{2}(\mathrm{biq})\right]^{+}\left(\mathrm{PF}_{6}{ }^{-}\right)$. The energy gaps estimated by cyclic voltammetry for complex 4 
Table 2. Summary of Physical Properties of Complexes 1-4

\begin{tabular}{|c|c|c|c|c|c|c|}
\hline \multirow[b]{2}{*}{ complex } & \multicolumn{3}{|c|}{$\lambda_{\max , P L}(\mathrm{~nm}), \Phi, \tau(\mu \mathrm{s})^{a}$} & \multirow[b]{2}{*}{$E_{1 / 2}^{0 \mathrm{x}}(\mathrm{V})^{f}$} & \multirow[b]{2}{*}{$E_{1 / 2}^{\mathrm{ed}}(\mathrm{V})^{t}$} & \multirow[b]{2}{*}{$\Delta E_{1 / 2}(\mathrm{~V})$} \\
\hline & solution ${ }^{b}$ & neat film or host-guest film & film with $B M I M+\left(\mathrm{PF}_{6}^{-}\right)^{c}$ & & & \\
\hline 1 & $499,0.46,0.71$ & $513,0.20,0.36$ & $504,0.22,0.42$ & $+1.29^{g}$ & $-1.70^{i}$ & 2.99 \\
\hline 2 & $497,0.66,0.85$ & $507,0.22,0.51$ & $498,0.26,0.56$ & $+1.28^{g}$ & $-1.72^{i}$ & 3.00 \\
\hline 3 & $491,0.54,0.73$ & $491,0.28,0.55$ & $488,0.30,0.74$ & $+1.20^{h}$ & $-1.79^{i}$ & 2.99 \\
\hline 4 & $656,0.20,0.75$ & $672,0.09,0.33$ & & $+0.86^{i}$ & $-1.37^{i}$ & 2.23 \\
\hline $\mathbf{4}(0.2 \mathrm{wt} \%) / \mathbf{3}$ & & $(493,606), 0.26,\left(0.49^{d}, 1.54^{e}\right)$ & $(488,603), 0.29,\left(0.54^{d}, 1.68^{e}\right)$ & & & \\
\hline $4(0.4 \mathrm{wt} \%) / 3$ & & $(493,614), 0.27,\left(0.38^{d}, 1.52^{e}\right)$ & $(488,612), 0.28,\left(0.47^{d}, 1.68^{e}\right)$ & & & \\
\hline
\end{tabular}

${ }^{a}$ At room temperature. ${ }^{b}$ Measured in $\mathrm{CH}_{2} \mathrm{Cl}_{2}\left(10^{-5} \mathrm{M}\right) .{ }^{c}$ Films containing $19 \mathrm{wt} \% \mathrm{BMIM}^{+}\left(\mathrm{PF}_{6}{ }^{-}\right) .{ }^{d}$ Measured at $480 \mathrm{~nm} .{ }^{e}$ Measured at $650 \mathrm{~nm} .{ }^{f}$ Potential vs ferrocene/ferrocenium redox couple. ${ }^{g} \mathrm{TBAPF}_{6}(0.1 \mathrm{M})$ in acetonitrile. ${ }^{h} \mathrm{TBAPF}_{6}(0.1 \mathrm{M})$ in $\mathrm{CH}_{2} \mathrm{Cl}_{2} .{ }^{i} \mathrm{TBAP}(0.1 \mathrm{M})$ in acetonitrile.

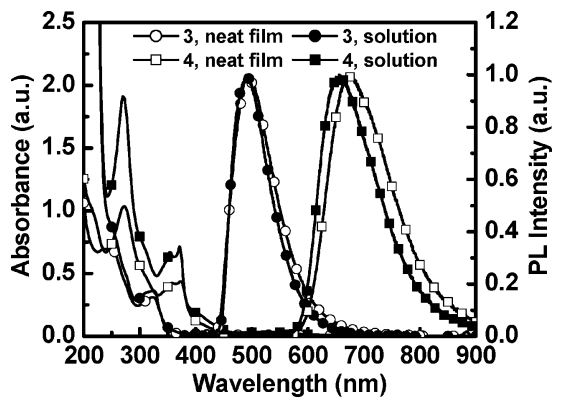

Figure 1. Absorption (left axis) and PL spectra (right axis) of complexes 3 and 4 in dichloromethane solutions $\left(10^{-5} \mathrm{M}\right)$ and in neat films.

$\left(2.23 \mathrm{eV}\right.$, Table 2) and $\left[\mathrm{Ir}(\mathrm{ppz})_{2}(\mathrm{biq})\right]^{+}\left(\mathrm{PF}_{6}{ }^{-}\right)(2.45 \mathrm{eV})^{4 \mathrm{~m}}$ are consistent with the photophysical observation. For $\left[\mathrm{Ir}(\mathrm{ppz})_{2}(\mathrm{biq})\right]^{+}-$ $\left(\mathrm{PF}_{6}{ }^{-}\right)$, the HOMO and the LUMO are predominantly localized on the ppz and biq ligand, respectively. ${ }^{4 \mathrm{~m}}$ The smaller energy gap of complex $\mathbf{4}$, which also contains biq, is thus associated with destabilization of HOMO in replacing ppz with ppy.

Cyclic voltammetry (CV) was used to probe the electrochemical properties of these complexes. The results are summarized in Table 2. For complexes $\mathbf{1}, \mathbf{2}$, and $\mathbf{3}$, one reversible oxidation potential was detected, which can be attributed to the oxidation occurring on the Ir center. Interestingly, the electronic nature of ancillary daf ligands plays a subtle role on the oxidation potential. For example, complex $\mathbf{3}$ containing a more electro-rich dedaf ligand shows a lower oxidation potential (1.20 V) compared with those of complexes $\mathbf{1}(1.29 \mathrm{~V})$ and $\mathbf{2}(1.28$ V), both containing aryl substitutions on C9 of the diazafluorene ligand. On the other hand, the reduction capability of the diazafluorene ligand is presumably responsible for observed reversible reduction of complexes $\mathbf{1}, \mathbf{2}$, and $\mathbf{3}$. Thus, the Ir complex coordinated to an electron-rich dedaf ligand (complex 3) shows a higher reduction potential $(-1.79 \mathrm{~V})$ compared with those complexed to the aryl-substituted daf ligand [complexes $\mathbf{1}(-1.70 \mathrm{~V})$ and $\mathbf{2}(-1.72 \mathrm{~V})]$. These results thus suggest that the energies of frontier orbitals in these complexes can be subtly manipulated by tailoring the electronic properties of the auxiliary daf ligands. For the red complex 4, one reversible oxidation $(0.86 \mathrm{~V})$ and one reversible reduction $(-1.37 \mathrm{~V})$ were detected. The electron-rich character of the ppy ligand and the more $\pi$-delocalized biq ligand contribute to the higher oxidation and lower reduction, respectively, thus leading to a small energy gap of complex 4.

Emission properties of the $\mathbf{3 / 4}$ host-guest films are also summarized in Table 2. Figure 2 depicts the PL spectra of the host - guest films with different guest $\mathbf{4}$ concentrations ( 0.4 and $0.2 \mathrm{wt} \%)$ with or without the presence of $\mathrm{BMIM}^{+}\left(\mathrm{PF}_{6}{ }^{-}\right)(19$ wt \%). In general, with the presence of $\mathrm{BMIM}^{+}\left(\mathrm{PF}_{6}{ }^{-}\right)$, both the host and guest exhibit longer excited-state lifetimes, and

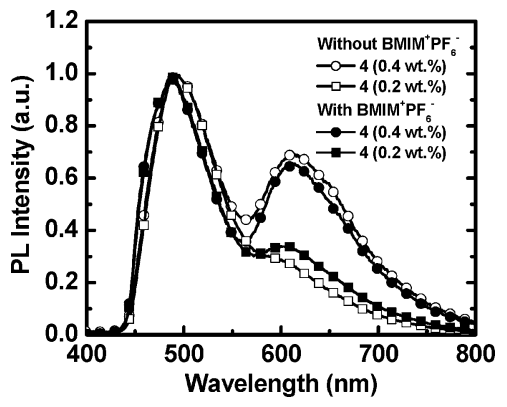

Figure 2. PL spectra of host-guest films containing different guest concentrations $\left(0.4\right.$ and 0.2 wt \%) without and with $\mathrm{BMIM}^{+}\left(\mathrm{PF}_{6}{ }^{-}\right)$ (19 wt \%).

Table 3. Summary of LEC Device Characteristics

\begin{tabular}{ccccccc}
\hline $\begin{array}{c}\text { bias } \\
(\mathrm{V})\end{array}$ & $\mathrm{CIE}(x, y)^{\mathrm{a}}$ & $\left.\mathrm{CR}\right|^{\mathrm{a}}$ & $\begin{array}{c}t_{\max }{ }^{b} \\
{[\mathrm{~min}]}\end{array}$ & $\begin{array}{c}L_{\max }{ }^{\mathrm{C}} \\
{\left[\mathrm{cd} / \mathrm{m}^{2}\right]}\end{array}$ & $\begin{array}{c}\eta_{\text {ext,max }}, \eta_{\mathrm{L}, \max }, \\
{[\%, \mathrm{~cd} / \mathrm{A}, \mathrm{Im} / \mathrm{W}]}\end{array}$ & $\begin{array}{c}t_{1 / 2}{ }^{e} \\
{[\mathrm{~h}]}\end{array}$ \\
\hline 2.9 & $(0.45,0.40)$ & 81 & 240 & 2.5 & $4.0,7.2,7.8$ & 8.9 \\
3.1 & $(0.37,0.39)$ & 80 & 60 & 18 & $3.4,6.1,6.2$ & 1.3 \\
3.3 & $(0.35,0.39)$ & 80 & 30 & 43 & $3.3,5.8,5.5$ & 0.4 \\
\hline
\end{tabular}

${ }^{a}$ Evaluated from the EL spectra. ${ }^{b}$ Time required to reach the maximal brightness. ${ }^{c}$ Maximal brightness achieved at a constant bias voltage. ${ }^{d}$ Maximal external quantum efficiency, cd/A, and power efficiency achieved at a constant bias voltage. ${ }^{e}$ The time for the brightness of the device to decay from the maximum to half of the maximum under a constant bias voltage.

the PLQYs are slightly raised (Table 2), indicating the role of $\mathrm{BMIM}^{+}\left(\mathrm{PF}_{6}{ }^{-}\right)$in suppressing intermolecular interactions. ${ }^{4 \mathrm{q}} \mathrm{As}$ shown in Figure 2, white emission of different blue-green and red compositions can be achieved by adjusting the guest concentration. Raising the guest concentration effectively enhances the host-to-guest energy transfer, accompanied by shorter lifetimes of the host emission (Table 2). The host-guest film containing $0.4 \mathrm{wt} \% \mathbf{4}$ (guest) shows white emission having CIE coordinates of $(x, y)=(0.34,0.37)$, which is rather close to the ideal equal-energy white $(x, y)=(0.33,0.33)$, and therefore was subjected to further EL studies.

The LECs have the structure ITO/emissive layer (100 nm)/ $\mathrm{Ag}(150 \mathrm{~nm})$, where the emissive layer contains host 3 (80.5 wt \%), guest 4 (0.4 wt \%), and $\mathrm{BMIM}^{+}\left(\mathrm{PF}_{6}{ }^{-}\right)(19.1$ wt \%). Their EL properties are summarized in Table 3. The EL spectra of the white LECs under various biases, along with the PL spectra for comparison, are shown in Figure 3a. It is noted that the peak wavelength of the blue component in the EL spectra is $488 \mathrm{~nm}$, which is one of the shortest EL wavelengths reported to date for LECs based on cationic transition metal complexes. Compared with PL, the relative intensity of the red emission with respect to the blue emission is larger in EL and increases as the bias decreases. Furthermore, the maximum current density of the host-guest device (with 0.4 wt \% guest) is lower than 

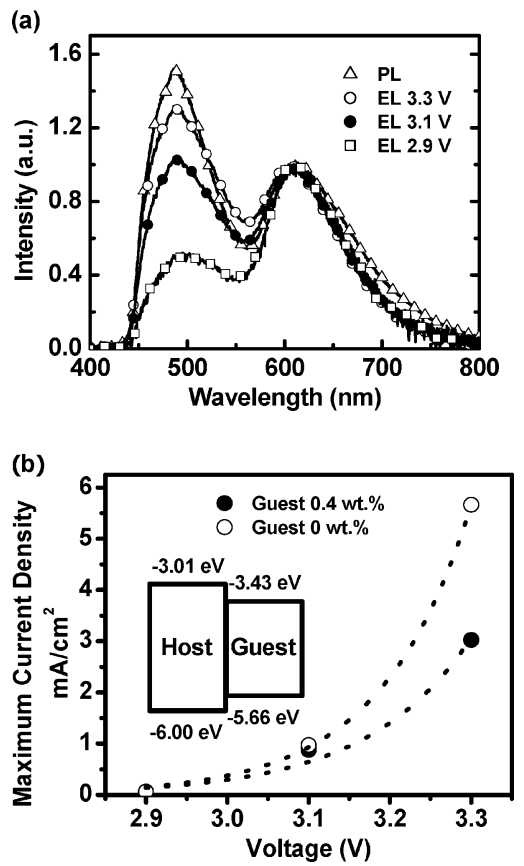

Figure 3. (a) The bias-dependent EL spectra of the white LECs compared with the PL spectrum and (b) maximum current density vs voltage characteristics for the host-guest ( $0.4 \mathrm{wt} \%$ doping concentration) and the host-only LECs. The inset shows the energy level diagram of the host and guest molecules.

that of the host-only device under same bias conditions (Figure $3 b)$. These results could be understood by energy level alignments of the host and guest (inset of Figure 3b). At lower biases, such energy level alignments favor carrier injection and trapping on the smaller-gap guest, resulting in direct carrier recombination/exciton formation on the guest (rather than host-guest energy transfer). Therefore, larger fractions of guest emission are observed at lower biases. Nevertheless, white emission with CIE coordinates of $(0.35,0.39)$ could be achieved at higher biases and brightnesses. Also, the present white LEC exhibits a rather high CRI (up to 80), which is an important characteristic for solid-state lighting.

Figure $4 \mathrm{a}$ shows the time-dependent brightness and current density under constant biases of 2.9-3.3 V for the white LEC. After the bias was applied, the current first rose and then stayed rather constant. On the other hand, the brightness first increased with the current and reached the maxima of $2.5,18$, and 43 $\mathrm{cd} / \mathrm{m}^{2}$ under biases of $2.9,3.1$, and $3.3 \mathrm{~V}$, respectively. The brightness then dropped with time with a rate depending on the bias voltage (or current). ${ }^{4}$ Corresponding time-dependent EQEs and power efficiencies of the same device are shown in Figure $4 \mathrm{~b}$. When a forward bias only was applied, the EQE was rather low due to poor carrier injection. During the formation of the doped regions near electrodes, the capability of carrier injection was improved and the EQE thus rose rapidly. The peak EQEs, cd/A, and power efficiencies at 2.9, 3.1, and $3.3 \mathrm{~V}$ are $(4.0 \%, 7.2 \mathrm{~cd} / \mathrm{A}, 7.8 \mathrm{~lm} / \mathrm{W}),(3.4 \%, 6.1 \mathrm{~cd} / \mathrm{A}, 6.2$ $\mathrm{lm} / \mathrm{W})$, and $(3.3 \%, 5.8 \mathrm{~cd} / \mathrm{A}, 5.5 \mathrm{~lm} / \mathrm{W})$, respectively. The drop of efficiencies and brightness after reaching the peak value, as commonly seen in solid-state LECs, ${ }^{3,4}$ may be associated with a few factors. Before the current reaches a steady value, the carrier recombination zone may keep moving closer to one electrode due to discrepancy in electron and hole mobilities, which would induce exciton quenching. Further, the decrease
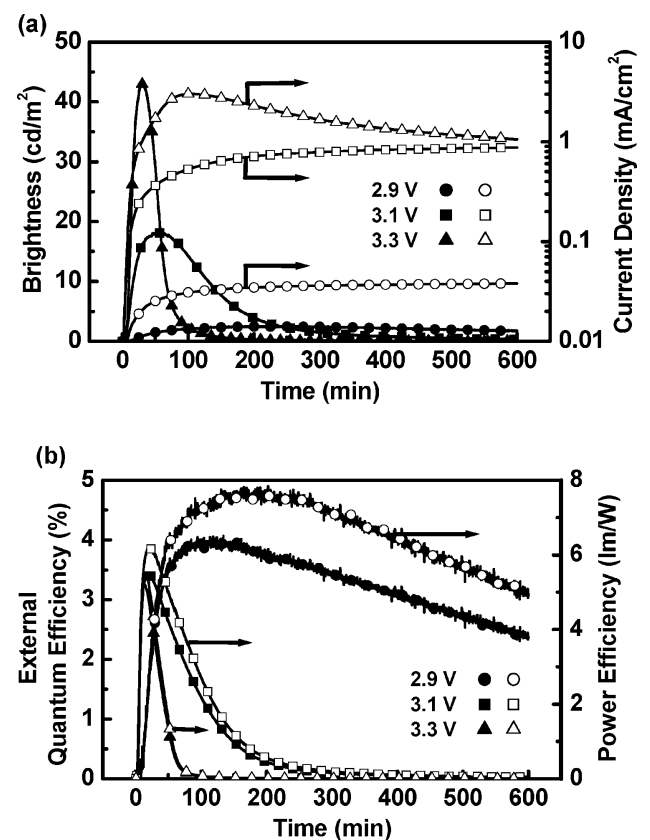

Figure 4. (a) Brightness (solid symbols) and current density (open symbols) and (b) external quantum efficiency (solid symbols) and power efficiency (open symbols) as a function of time under a constant bias voltage of 2.9$3.3 \mathrm{~V}$ for the white LEC.

in brightness and efficiencies under a constant bias was irreversible and thus may be rationally associated with the degradation of the emissive material during the LEC operation. ${ }^{4 \mathrm{~d}}$ Furthermore, although the device clearly gave white emission, the brightness currently achieved is still limited. The limited brightness is to some degree associated with the relatively lower emission quantum yields (e.g., hosts and guests). By further improving the materials to get higher emission efficiencies, it is believed that the brightness can be much further enhanced.

Peak brightness and turn-on time (the time required to reach the maximal brightness) as a function of bias voltage for white LECs are shown in Figure 5a. An electrochemical junction between $\mathrm{p}$ - and n-type doped layers of LECs is formed during device operation. As revealed in previous studies, ${ }^{4 \mathrm{f}}$ as bias voltage increases, the junction width decreases due to extension of doped layers, consequently leading to higher current density (Figure 4a) and higher brightness (Figure 5a). The higher electric field in the device also accelerates redistribution of mobile ions, which facilitates formation of ohmic contacts with the electrodes. Thus, operation of LECs under higher bias voltages fastens the device response (Figure 5a). However, higher brightness and faster response are obtained at the expense of device stability. As shown in Figure 5b, peak EQE and device lifetime (the time for the brightness of the device decaying from the maximum to half of the maximum under a constant bias voltage) of the white LECs deteriorate under higher bias voltages. It may be associated with the higher electric field or current density accelerating degradation (e.g., multiple oxidation and subsequent decomposition $)^{4 \mathrm{k}}$ of the emissive cationic transition metal complexes. Similarly, previous studies showed that modulation of the electronic properties of the emissive layer of LECs by different additives has significant influence on the device lifetimes. ${ }^{4 \mathrm{~b}, \mathrm{c}}$ For instance, blending an inert polymer ${ }^{4 \mathrm{c}}$ into the emissive layer of LECs improves device lifetimes, while adding electrolytes $^{4 \mathrm{~b}}$ shortens device lifetimes. Blending with an inert polymer reduces the electronic conductivity of the emissive layer 
(a)

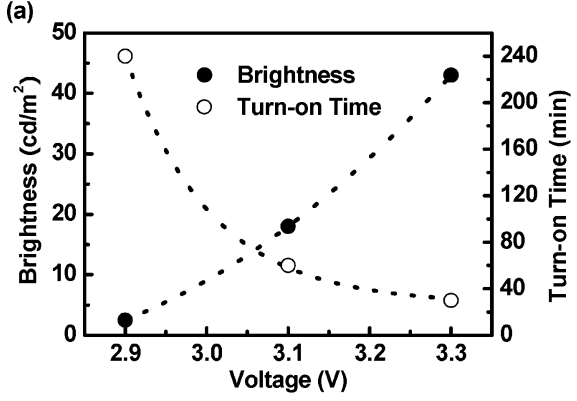

(b)

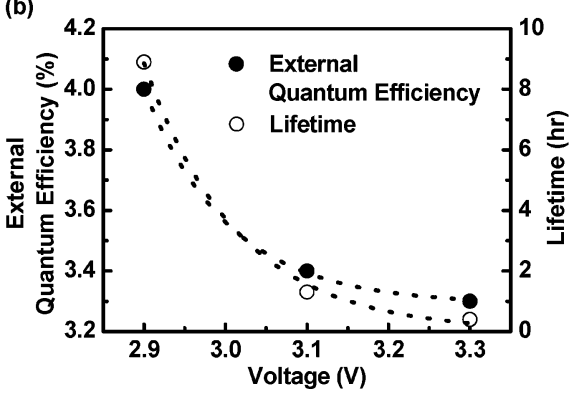

Figure 5. (a) Peak brightness (solid symbols) and turn-on time (open symbols) and (b) peak external quantum efficiency (solid symbols) and lifetime (open symbols) as a function of bias voltage for the white LECs.

and thus lowers the current density. In contrast, additional mobile ions provided by the electrolyte increase the doping level and conductivity, resulting in higher current density. A few recent reports ${ }^{4 \mathrm{r}, \mathrm{s}}$ have also revealed that electrical driving of LECs based on cationic transition metal complexes induces oxobridged dimers, which effectively quench electroluminescence. Despite these sporadic studies, detailed degradation mechanisms of the LECs based on cationic transition metal complexes remain unclear, and further studies are still needed to achieve practical device lifetimes.

In summary, we have reported the synthesis and characterization of efficient cationic blue-green-emitting complexes, $\left[\operatorname{Ir}(\mathrm{dfppz})_{2}(\mathrm{dasb})\right]^{+}\left(\mathrm{PF}_{6}{ }^{-}\right)(\mathbf{1}),\left[\operatorname{Ir}(\mathrm{dfppz})_{2}(\mathrm{bmpdaf})\right]^{+}\left(\mathrm{PF}_{6}{ }^{-}\right)(\mathbf{2})$, and $\left[\operatorname{Ir}(\mathrm{dfppz})_{2}(\mathrm{dedaf})\right]^{+}\left(\mathrm{PF}_{6}{ }^{-}\right)(\mathbf{3})$, and a red-emitting complex, $\left[\operatorname{Ir}(\mathrm{ppy})_{2}(\mathrm{biq})\right]^{+}\left(\mathrm{PF}_{6}{ }^{-}\right)(4)$. By rationally combining these emitters in the host-guest systems, for the first time, we successfully generated white EL from solid-state LECs based on host-guest cationic iridium complexes, with decent color performances and efficiencies. These results suggest that white LECs based on host-guest cationic transition metal complexes may be a promising alternative for solid-sate lighting technologies.

Acknowledgment. We are grateful for financial support from National Science Council, Ministry of Education, and Ministry of Economic Affairs of Taiwan.

Supporting Information Available: CIF files of complexes 3 and 4. This material is available free of charge via the Internet at http://pubs.acs.org.

JA076051E 